\title{
The Surgical Management of Foot Deformities in Leprosy
}

\author{
W. M. LENNOX, B.sG., F.R.G.s.(ENG.), F.R.G.s.(EDIN.) \\ Department of Orthopaedics, C.M.C. Hospital, Vellore
}

Trophic ulcers (or 'plantar ulcers') may occur on structurally normal but anaesthetic feet, and these have been called the 'dynamic type' ulcer, by Price ( 1963 ). They occur on pliant feet, heal rapidly with rest, and can be prevented by appropriate shoes. On the other hand, the presence of a deformity may promote recurrent and intractable ulcers even when shoes are worn. Ulcers associated with a fixed deformity have been called by Price the 'static type'.

Untreated, these feet ultimately undergo total destruction, and may be considered for amputation. Lately, however, it has become clear that many of these feet can be salvaged and made useful again by corrective surgery and appropriate shoes. The classification of foot mutilations resulting from leprosy has been the subject of a separate communication (Lennox). Five groups of deformity were described, and their main features discussed. It is the purpose of this paper to review those surgical methods which in the author's experience are useful in managing deformities which have a specific relationship to ulcers.

THE SURGICAL TREATMENT OF GROUP I DEFORMITIES

\section{Dropped foot}

In a study of plantar pressures during walking using pressure sensitive transducer discs, it was shown that in the dropped foot there was an accentuation of pressure along the antero-lateral border of the foot (Bauman et al.). It is here that the characteristic ulcers of the deformity occur. It should be one of the objects of surgical treatment to eliminate this pressure accentuation, and hence to remove an undoubted predisposing factor. A simple method of detecting this pressure in dropped foot is to take foot-print using an inked rubber mat (technique described by Price, I963), on which accentuated pressures show as areas of heavy inking. A similar impression taken after operation will show the extent of pressure reduction obtained. Most examples of foot drop are complete, but in some instances, sparing of the peronei is seen. With strong peronei and absent tibialis anterior, the lateral balance of the foot is upset in favour of the evertors, and pressures along the antero-medial border of the foot are accentuated, causing a tendency for ulcers to occur here. Typically, absorption of the medial ray of the foot then occurs. The pattern of absorption of a damaged foot and the location of ulcer scars has an important bearing on the choice of foot drop correction.

Equinus deformities are easily corrected by a tendon transfer, and the operation of choice is transplantation of the tibialis posterior tendon into the dorsum of the foot. One of the principles of reconstructive surgery is that no tendon transfer should be carried out until the pattern of paralysis has become stable. Thus, timing of the transfer is of importance. It is not always easy to be satisfied on this point in cases of lateral popliteal paralysis. Lateral popliteal paralysis can recover and in cases of sudden or rapid onset (usually borderline cases), the prognosis may be comparatively favourable if the knee is promptly immobilized in a full length leg plaster. However, experience shows that recovery is unlikely to occur if six or more months have elapsed since the onset of the paralysis so that it is our custom not to operate before this time, but to operate as soon after six months as possible. Very occasionally, a spontaneous recovery of the anterior tibial muscle has been seen after tibialis posterior transfer, but this causes no untoward effect providing the plantar flexors are intact.

The presence of active peronei may be regarded as evidence that a stable pattern of paralysis has not been reached. This may be true, but a period of observation will show 

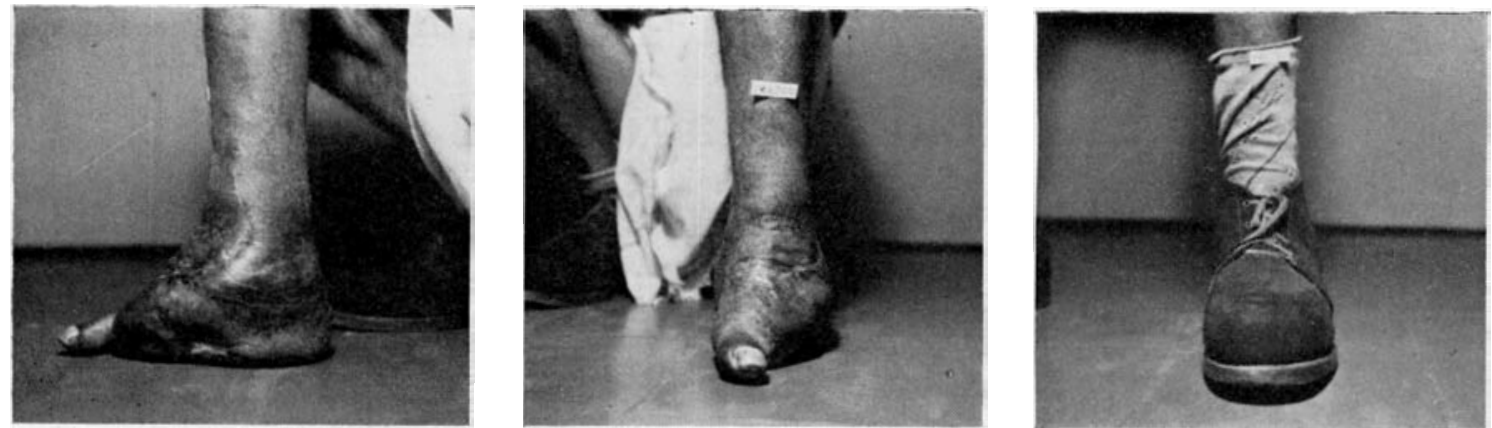

FIG I A foot remnant rendered stable by Pantalar Arthrodesis, and the final appearance wearing a boot.

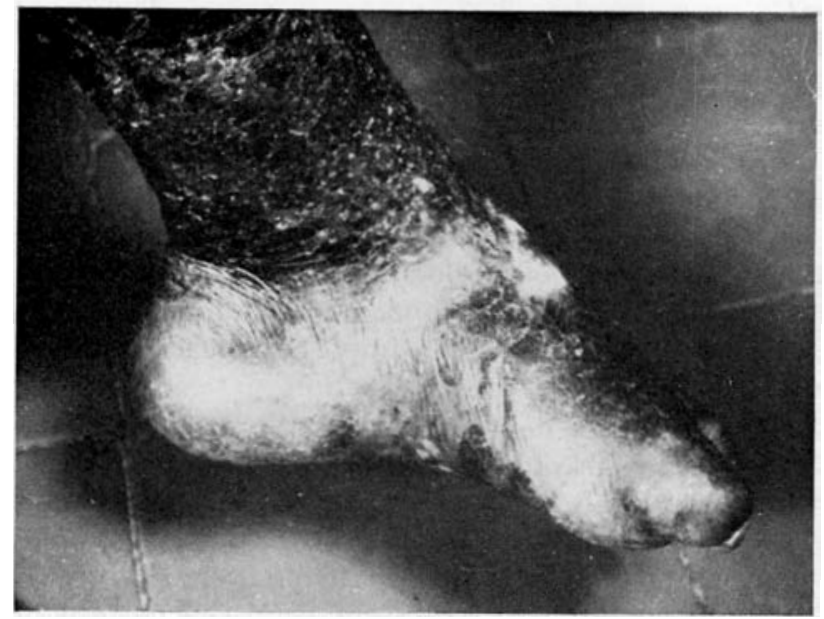

FIG 2 A fixed Equino-varus foot, showing the scarred area on the lateral border, and the soft tissue contracture behind and medial to the ankle. This foot requires Tibio-calcaneal fusion.
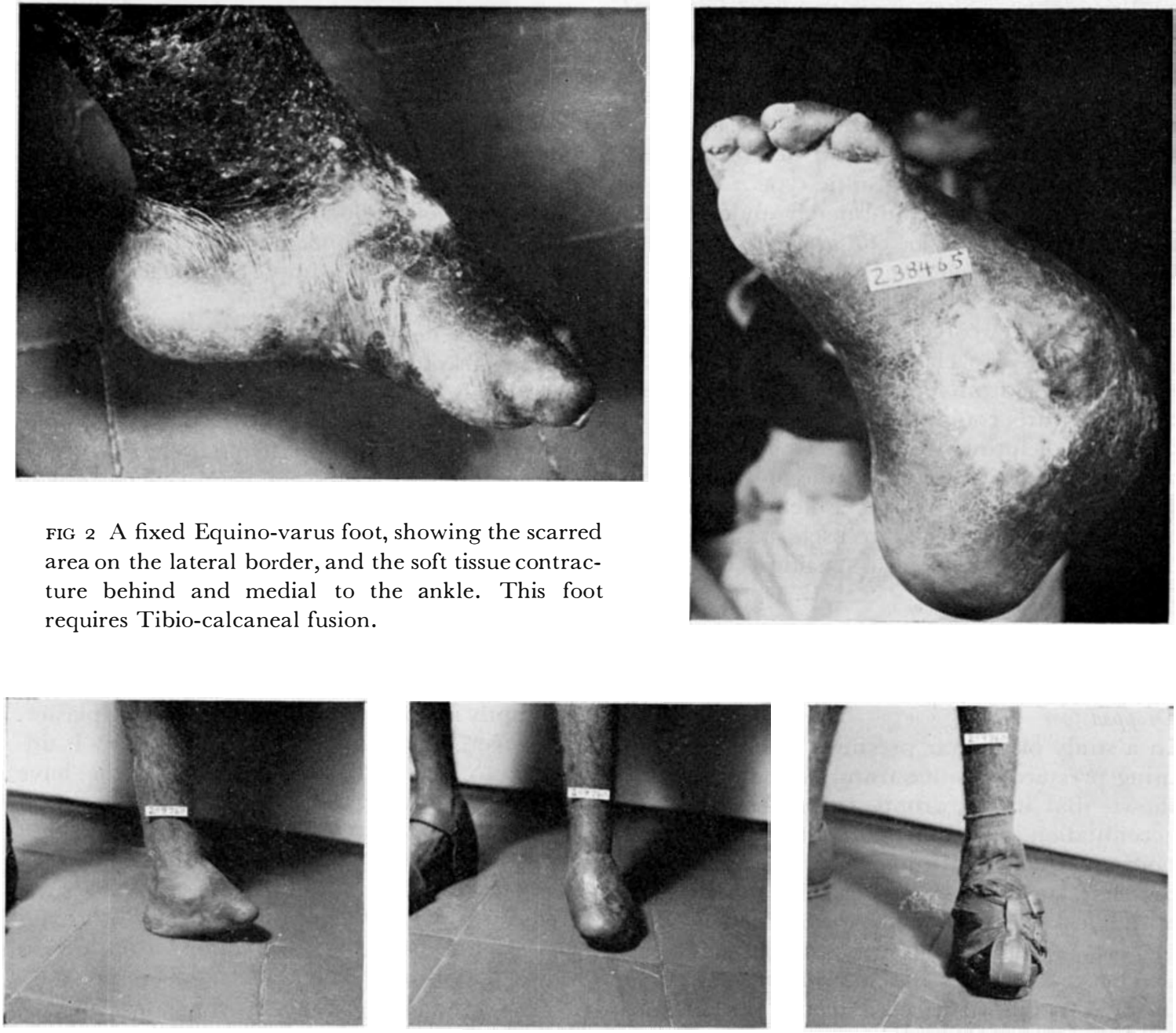

FIG 3 An Equino-varus foot remnant rendered stable by 'Tibio-calcaneal fusion, and the final appearance in a sock and sandal. 

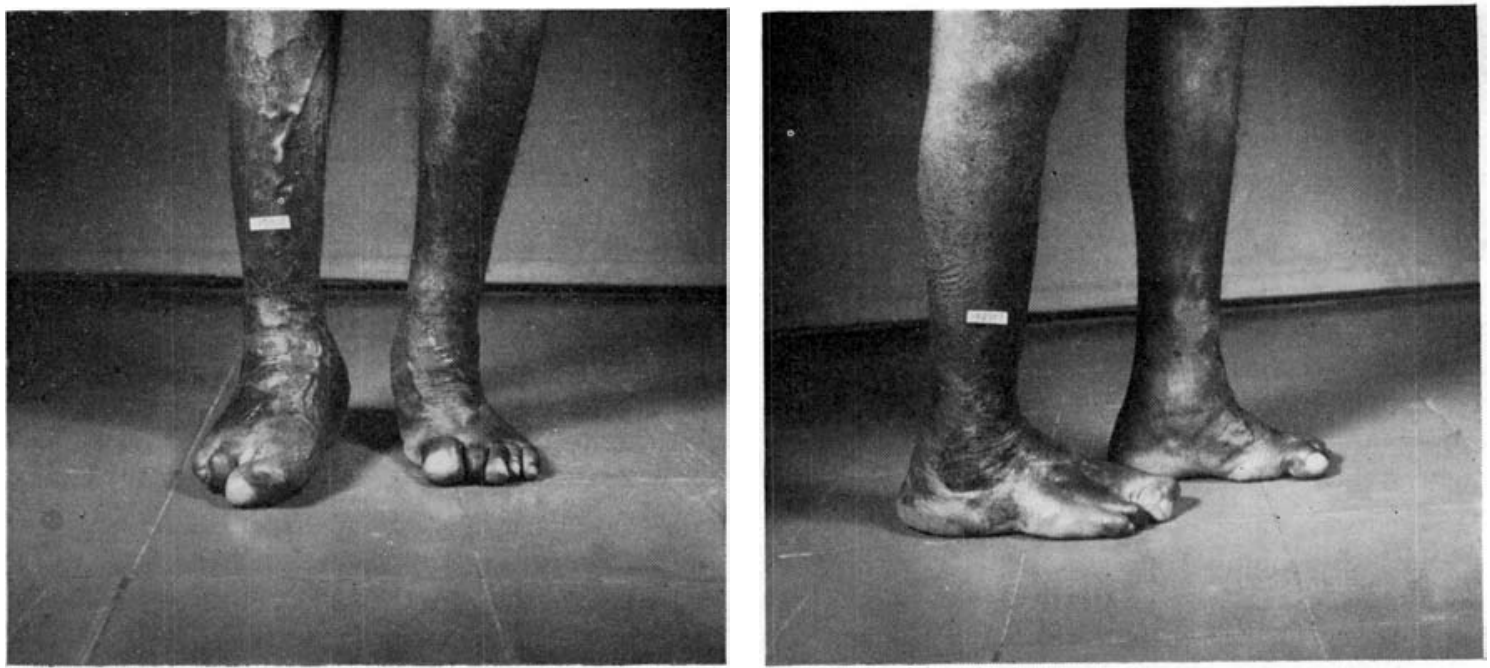

FIG 4 A Neuropathic right foot rendered stable by Triple arthrodesis.

whether recovery is occurring, or whether the paralysis is progressing. If there is evidence of recovery, then conservative management is indicated (a toe raising spring harness); if the peronei are weakening, then surgery should be undertaken as if the case were one of complete foot drop. In patients receiving regular medication, if the strength of the peronei appears to be constant, surgery is undertaken, but a different type of correction is selected. It is undesirable to defer foot drop surgery for too long pending a decision on the course being followed by the peronei. Patients dislike wearing foot lifting harness for longer than necessary, and a delay in surgery delays their ultimate rehabilitation.

How soon after the healing of an ulcer on a dropped foot should one carry out a surgical correction? There is no hard or fast rule, but it is our custom to wait for four weeks, and this system has worked well in practice.

The operation of tibialis posterior transfer as applied to leprosy patients has been well described (Gunn and Molesworth i 957), (Selvapandian and Brand I959), and it is not necessary to repeat the descriptions. It is now our custom to employ a modification of the interosseous route, and to attach the transfer to the lateral cuneiform without disturbing the bone. This is to ensure a good lift to the lateral border of the foot, and this, combined with removal of the tibialis posterior from its rôle of invertor, eliminates the accentuated lateral pressures. Lateral balance is usually good. If later a valgus deformity develops, it is treated by a subtalar fusion of Grice-Green (1952) type.

In the presence of strong stable peronei, either a circumtibial tibialis posterior transfer or an interosseous transfer with a more medial insertion is required, so that lateral balance of the foot may be achieved. If the peronei later becomes paralysed, it may become necessary to shift the insertion of the transfer laterally, to tenodese the peroneus brevis to the transfer, or to perform an arthrodesis. Because, one can never be sure in leprosy whether apparently strong peronei will later become paralysed or not; these muscles are not used as prime dorsiflexors.

A successful foot drop correction will restore active dorsiflexion to the foot, restore the gait to normal, give a laterally balanced foot, and a normal distribution of plantar pressures. As these feet remain anaesthetic, they also require a simple shoe. Shoes alone do not afford protection against ulcers on a dropped foot.

\section{Claw Toes}

A number of operations have been described for this deformity, and before the toes become stiff correction by multiple transfer of the flexor digitorum longus tendons into the extensors is a simple procedure, and gives good results. 
Taylor (I95I) reviews the operation and its good results in 68 (non-leprosy) cases. The results in leprosy are equally gratifying. The objects of the operation are (a) to correct the alignment of the toes so that the pulps came into contact with the ground; (b) to replace the fibrofatty pad beneath the metatarsal heads. This may be difficult once ulceration has occurred and the scars tether the skin to the underlying bone. There is no doubt that the time for correction is before ulcers have occurred. This simple operation is not used often enough in the early stages when the deformity is easily corrected.

Once the metacarpophalangeal joint begins to subluxate and the deformity begins to pass into group II, the difficulties of a successful correction are increased. Manual toe stretching by a physiotherapist is a useful but much neglected way of correcting a mild subluxation, after which a tendon transfer will suffice. A preliminary subcutaneous extensor tenotomy may help; but in many cases a dorsal capsulotomy and extensor lengthening are needed at the time of correction. Longstanding cases usually have a skin contracture as well so that a skin graft may have to be employed to cover a defect created over the dorsum of the forefoot. If it appears likely that a claw toe correction will involve a skin graft, extensor tenotomy, metacarpophalangeal capsulotomy, and tendon transfers or proximal interphalangeal arthrodesis, then amputation of the clawed toes is a better procedure (Nissen). This may, in any case, be the only choice for rigidly clawed toes perched dorsally upon the metatarsal necks, and subject to frequent shoe blisters. This operation has been employed for the ischaemic clawed toes in diabetes (Oakley et al) in Rheumatoid Arthritis, Hallux Valgus and Pes Cavus (Flint and Sweetnam). In many of these conditions, pain is the crippling symptom. This prevents the patients from walking and thus from getting ulcers or blisters. In leprosy, there is no pain but the rationale of operation is similar. By removing the subluxated toe, the displaced fibro-fatty pad is allowed to drop back under the metatarsal head. This will only happen if there are no scars between the skin and the underlying bone. This operation therefore is for the relatively unscarred forefoot. In sensitive foot, the operation causes no disability though patients find it easier to balance in a normal shoe with a filled in toe. In the pressure disc study (Bauman et al) it was found that the feet shortened by trophic ulceration (and therefore, scarred) loss of toe function resulted in accentuation of anterior pressures. But, if the anterior pad drops back into place under the metatarsal heads, then sufficient tissue reserve becomes available to withstand the stresses of ordinary activity. Nevertheless, in leprosy cases, a moulded arch insole or a metatarsal bar shoe should be given in order to spare the metatarsal heads as much as possible from the extra pressure occasioned by loss of the toes.

When there is scarring under the metatarsal heads, the following operation, which is useful in Rheumatoid Arthritis deformity, may be considered. Through an anterior incision just in front of the weight bearing surface, all the metatarsal heads are excised. In leprosy feet, this is useful where there are multiple ulcer scars under the distal metatarsal arch and where the metatarsal heads are particularly prominent. Patients agree to this operation more readily than to total amputation and the results are functionally satisfactory, apart from the hazard of friction from shoes against anaesthetic toes.

An amputation through the metatarsals (Lisfranc) combines the advantages of removal of anaesthetic deformed toes with excision of bony points, and is required for cases with extensive anterior plantar scarring. A level of bone section can be selected which allows easy closure with dorsal and plantar flaps, and a substantial pad of plantar pulp can be arranged to underly the front of the bony skeleton. Unfortunately we have not been able to employ this excellent operation as often as we have advised it, due to willingness of many of our patients to sacrifice their toes, whatever the risk of ulcers.

THE SURGICAL TREATMENT OF GROUP II DEFORMITIES

The important features of this group of deformities have already been described. In addition to reduction and deflection of plantar surface, marginal scarring and concentration of weight bearing onto small areas, it has been shown that in the shortened foot, anterior pressures at 'push off' are significantly higher than those in feet of normal length (Bauman et al). 
The aims of treatment may be stated thus:

(a) To restore all the available plantar surface to its weight bearing function;

(b) to relieve scarred areas from weight bearing and shearing stresses;

(c) to correct and stabilize the deformity by bone surgery or tendon transfer;

(d) to modify excessive anterior push off pressures and protect the foot by suitable shoes.

These principles may be applied to the deformities of this group as follows:

\section{The shortened equinus foot}

This foot ulcerates anteriorly. The deformity is fixed by shortening of the tendo achilles, and contracture of the ankle capsule. The tibialis posterior is usually present and active. Correction can be obtained by a single or two-stage soft tissue operation, viz., lengthening of tendoachilles, posterior capsulotomy and tibialis posterior transfer. The management is then that of a tibialis posterior transfer. It is not common to obtain a large range of movement after this operation but this does not matter as long as the foot is maintained at about $90^{\circ}$.

Difficulty is sometimes encountered due to the extreme shortening of the structures behind the ankle joint, notably the skin, which in this type of case is usually contracted and lacking in resilience. The procedure to be adopted depends on whether there is hope of movement at the ankle joint or not (examination of an X-ray is helpful). If it is felt that movement can be expected, then all the posterior structures should be drastically lengthened, and the foot brought up at least to a right angle. In order to achieve this, a large flap of calf skin may have to be mobilised in continuity with the heel, and induced to slide downwards as the foot is dorsiflexed. The defect over the upper calf is then grafted with split thickness skin. At a second stage the tibialis posterior is transferred onto the dorsum of the foot to provide active dorsiflexion.

This procedure is not justified if there is no hope of ankle function afterwards. It is then better to carry out a pantalar arthrodesis (Fig. I). Even this may not be feasible when extreme posterior soft tissue contracture does not allow the foot to be brought up to a right angle. In these circumstances we excise the talus, and fuse the lower end of the tibia to the calcaneum.

\section{The shortened plantigrade foot}

This requires special shoes to modify the high anterior pressures at 'push off'. On the basis of the pressure disc study (Bauman et al) rigid sole rocker shoes were recommended. Unfortunately these have proved to be unsatisfactory in practice, and we now emply modified metatarsal bar shoes or ankle boots.

\section{The inverted foot and the shortened inverted foot}

Simple inversion is corrected by means of a subtalar arthrodesis with excision of a laterally based wedge. It is important to aim at over correction rather than exact correction, as the position of the finished foot is often not as good as appeared on the operating table.

In many cases there is an associated foot drop with an active tibialis posterior. The choice of operation again depends upon whether a mobile ankle joint is expected or not. If surgery is undertaken with hope of a functioning ankle, then a two-stage correction is undertaken, as follows:

(i) Triple arthrodesis with resection of an appropriate wedge for correction of the inversion.

(ii) Lengthening of the tendo-Achilles, posterior capsulotomy of ankle, and tibialis posterior transfer.

If there are degenerative changes in the ankle joint, or other reasons for supposing that active dorsiflexion may not be achieved then a pantalar arthrodesis is carried out, and the anterior lever of the foot is shortened.

The fixed, inverted, equinus foot is often more difficult to correct than might be expected. The reason for this is that the soft tissues on the concave side of the deformity have contracted and become fibrosed, and not infrequently joint surfaces are the seat of fibrous ankylosis (Fig. 2). After excision of a wedge based upon the convex side of the deformity, the contracted soft tissues on the medial side may be tough enough to prevent closure of the wedge, even when the apex of the wedge is of significant thickness and extends cleanly through to the medial side of the bone. Experiences of this kind with the severe fixed deformity have led us to advise excision of the talus and tibio-calcaneal fusion as the operation of choice. Some length is sacrificed, but the contracted tissues are relaxed sufficiently to permit alignment of the foot on the tibia at $90^{\circ}$ and without inversion or eversion (Fig. 3). 
THE SURGICAL TREATMENT OF GROUP III DEFORMITIES

These are deformities due to neuropathic destruction of bones and joints. The mechanism of initiation of breakdown of these feet is not clearly worked out. An extensive study of paralytic feet resulting from poliomyelitis enabled Grice to state that loss of support to the head of the talus appeared to be the cardinal cause of breakdown of the arch in these feet, and that breakdown of the support was initiated by the valgus posture consequent upon weight bearing on the unsupported foot. In valgus, the talus slides forwards and medially relative to the calcaneum so that the head loses most of its bony support, and relies more on the calcaneonavicular ligament. This movement of the talus is accentuated by (i) the upward and backward pull of strong calf muscles on the calcaneum; (ii) the forward and downward thrust imparted to the talus by body weight; (iii) loss of tibialis posterior and tibialis anterior ( $1 \mathrm{n}$ polio). In leprosy, tibialis posterior is spared, but support of the talo-navicular region is reduced by paralysis of the tibialis anterior, so that additional strains fall on the spring ligament. Normally, the plantar ligaments are strong enough to withstand the static strains placed upon them (Basajian and Stecko), but if the ligaments are softened or slackened from any cause, and the plantar and extrinsic foot muscles are paralysed then the ligaments may be expected to stretch. When this happens, the talar head drops, the lines of thrust and the mechanics of the midtarsal and other joints will be altered, and without the protection of pain, joint damage and neuropathy may be expected to set in. Factors which would sof ten or damage plantar ligaments may be listed as follows:

(I) Septic arthritis or osteomyelitis anywhere in the foot.

(2) Trophic ulceration anywhere in the foot.

(3) Painless fractures of the foot bones (relatively common occurrences in the anaesthetic foot).

All these conditions cause hyperaemia and oedema of the foot and therefore probably softening of the plantar ligaments. Being insensible of pain, the patient continues to walk, or is allowed to by his doctor. Then the ordinary stresses of walking, quite safe for normal ligaments, are likely to cause stretching of the odematous ligaments, and conditions are set for a neuropathy. The above conditions frequently occur in the leprosy foot, though this account can at present be little more than a working hypothesis. Nevertheless, it does raise the question of whether an extended period off weight bearing during acute ulceration, followed by a period of protected weight bearing, might not prevent the later development of neuropathy in leprosy feet. It is true that trophic ulcers in general heal while in a plaster cast, but it may be the patients who are most active in their plasters who later develop neuropathy.

Once neuropathy is established, arthrodesis is the operation of choice. It stabilises the foot and arrests an otherwise progressive condition (Fig. 4). Inlay grafts of cancellous bone have also been successful. Once it is certain that neuropathic degeneration has set in, operation should not be delayed. Surgery is easier and fusion more certain before bones become destroyed and sclerosed, and relationships distorted.

THE SURGICAL TREATMENT OF GROUP IV DEFORMITIES

Spurs on the undersurface of the calcaneum are frequently associated with an active ulcer or a scar, and sometimes with a smouldering osteomyelitis of the undersurface of the calcaneum. There may be associated sinuses. All these conditions are conveniently dealt with by an operation through a fishmouth incision round the heel margin. A flap is developed which reflects skin, heel pulp, plantar fascia and periosteum distally. The undersurface of the calcaneum is then trimmed flat with a sharp osteotome, cutting away all excresences and depressions. The flap is then allowed to fall back into place and the incision is sutured. Sloughing of the flap is an occasional complication. The risk of this is much reduced by carrying the incision along the lateral margin of the heel only, thus sparing the medial calcaneal vessels. Heel sinuses often connect with a focus of osteomyelitis on the undersurface of the calcaneum, and in these cases, the incision should be drained, and the sinus tracts either excised or curetted. A small scar in the flap can often be excised by an elliptical incision followed by primary suture if there is sufficient tissue laxity to allow closure without tension. On occasion small clean 
ulcers have been treated by this method without mishap.

THE BIZARRE HEEL DEFORMITY

This results from loss of calcaneum or talus, or parts of both, from osteomyelitis or neuropathy. With weight bearing, these deformities are unstable and progressive. It is not possible to apply a standardized operation to each variation but the principle of treatment is simple. Stabilization of the bony remains by whatever technique seems best suited to the particular case. The bones in these cases may often exhibit sclerosis, and the operator encounters tough interosseous fibrous tissue. As much as possible of this undesirable tissue should be removed. Fusion is most likely to occur with a compression technique, reinforced by cancellous bone shavings, or by a cancellous inlay or strut graft.

These operations never give a foot of glamorous appearance, but they usually result in a foot, which, with a carefully made shoe, and some care, can be walked on.

THE SURGICAL TREATMENT OF GROUP V DEFORMITIES

Calcaneus deformity is uncommon, and treatment to some extent depends upon the cause. If it results from excessive tensioning of a tibialis posterior transfer and there is sufficient passive plantar flexion at the ankle, it may be treated by lengthening the dorsiflexor and shortening the tendo-achilles. Some anterior skin contracture may be encountered and may necessitate a skin graft. All other cases require pantalar arthrodesis.

GENERAL MANAGEMENT OF FOOT

RECONSTRUCTION CASES

Many of these patients are anaemic and debilitated by long ulceration, sepsis, and malnutrition consequent upon inability to earn. Deformed feet are often fissured and orthopaedically very dirty. The legs may be covered with scabies and eczema. The case may present with open ulcers. It is the custom to admit these cases and to heal the ulcers by rest, elevation and Eusol dressings. In the meantime anaemia is corrected by oral or parenteral iron supplements, and the skin is brought into the best possible condition by scrubs and oil applications. Severe degrees of anaemia are transfused. A daily leg exercise class has helped to stimulate circulation and when the ulcers have healed, selected cases are given exercises on a bicycle ergometer, again with the object of developing the circulation. About six weeks after the last ulcer has healed, surgery of the underlying deformity is undertaken. In the case of foot drop, however, where the procedure is confined to the dorsum of the foot, surgery is undertaken after four weeks. These are arbitrary time periods with no special merit other than that they allow latent infection to either die away or manifest itself. They do not unduly delay the ultimate rehabilitation of the patient, and they have worked well in practice.

Where bony fusion is concerned, the most reliable results have been seen af ter employment of a compression technique, though an occasional troublesome pin track infection is seen. Our experience has been that plaster of Paris fixation alone, or internal fixation with Kirshner wires, does not promote sound bony union with predictable regularity. Staples have also been tried. They are convenient to use but are less likely to promote union unless the areas of cancellous bone in contact are large. The greater the degree of sclerosis present, the less the chance of sound bony union and we are using grafts of iliac cancellous bone more and more frequently. Post operatively, the leg is kept elevated in its padded cast. Next day straight leg raising and knee flexion exercises are commenced. After four weeks, the sutures are removed, after which X-rays are taken and the 'feel' of the arthrodesis is tested. In favourable cases, the compression pins may be removed, and a new padded plaster is applied. Weight bearing is allowed after six to eight weeks.

The arthrodesis will consolidate in 12 to 16 weeks, after which the foot is ready for shoe fitting.

\section{I S G US S I O N}

None of these operations will succeed unless a shoe is also worn, and surgery on the more mutilated feet has only become possible with the development of simple techniques for making moulded insoles. In many instances, the result is an unglamorous walking appendage, which, nevertheless, gives good functional service if properly cared for. This raises the question of 
selection of patients for salvage procedures. Only patients who have insight into their condition and into what is being attempted for them are likely to be benefited from these often intricate procedures.

The surgeon who sets out to salvage badly damaged feet must accept that he is operating on risky material, and must be prepared to lose an occasional foot. It is wise to explain this to the patient beforehand. Many of these feet would, in any case, be properly considered for amputation, so there is little to lose in attempting salvage if the patient is told at the beginning not to hope for too much. The difficulties of limb fitting to an anaesthetic stump are by no means as small as some writers suggest, so that a salvage procedure made possible by the assistance of the shoemaker, is by no means an unattractive alternative.

Hodges (1956) reported the use of a triple arthrodesis of Lambrinudi type on 15 cases of leprosy foot drop with acceptable results in all cases. We have had no experience of the use of this procedure, since all our cases have been treated by the simpler methods described. However, the method appears to be useful in cases of severe equinus where complete correction cannot be achieved by other forms of arthrodesis.

\section{S U M M A R Y}

The management of Leprosy foot deformities which are closely associated with trophic ulcers, is describcd. Emphasis is placed upon reconstructive and salvage procedures rather than upon amputation.

\section{A CKNOWLEDGEMENTS}

It is a pleasure to acknowledge the constant encouragement and guidance of $\mathrm{Dr}$ A. J. Selvapandian; also the help of $\mathrm{Mr}$ S. D. Sigamoney in the preparation of the photographs; and Mr B. V. Venkatesan's careful typing of the manuscript. This work has been made possible by funds made available by the Vocational Rehabilitation Administration, Washington, USA, and the British Leprosy Relief Association.

\section{Bibliography}

Basajan, J. v. and stecko, G. (1963). The Rôle of Muscles in Arch Support of the Foot. Journal of Bone and Joint Surgery, 45-A, I 184 .

BAUMAN, J. H., GIRLING, J. P. and BRAND, P. W. (I 963). Plantar Pressures and Trophic Ulceration. Fournal of Bone and Joint Sur gery, 45-B, $65^{2}$.

FLINT, M. and SWEETNAM, R . ( I 960). Amputation of all Toes. Journal of Bone and Joint Surgery, 42-B, 90.

GRICE, D. S. (1 952). An Extra Articular Arthrodesis of the Subastragular Joint for Correction of Paralytic Flat Feet in Children. Journal of Bone and Joint Sur gery, 34-A, 927.

GUNN, D. R. and molesworth, B. D. (I957). The use of Tibialis Posterior as a Dorsiflexor. Fournal of Bone and Joint Surgery, 39-B, 674.

Hodges, w.A. (1956). The Treatment of Deformities of the Foot in Leprosy. East African Medical Journal, 33, 302. Nissen, K. I. ( I 953). The place of Amputation of all Toes. Journal of Bone and Foint Surgery, 35-B, 488.

OAKLEY, w., CATTERALl, R. C. F. and MARTIN, M. M. (I956). Aetiology and Management of Lesions of the Feet in Diabetes. British Medical Journal, 953.

SElvapandian, A. J. and BRAND, P. W. (I 959). Transfer of the Tibialis Posterior in Foot-drop Deformities. Indian Journal of Surgery, 21, I $5 \mathrm{I}$.

TAYLOR, R. G. (I 95I). Treatment of Claw Toes by Multiple 'Transfers of Flexor into Extensor Tendons. Fournal of Bone and Foint Sur gery, 33-B, 539. 